\title{
GaN 2DEG Varactor-Based Impulse Suppression Module for Protection Against Malicious Electromagnetic Interference
}

\author{
CHIEN-FU SHIH,,$^{1,7}$ LIANN-BE CHANG (10, ${ }^{2,3,4,8}$ MING-JER JENG, ${ }^{1,3,9}$ YU- \\ LI HSIEH, ${ }^{1,5,10,11}$ YING-CHANG LI, ${ }^{6,12}$ and ZI-XIN DING ${ }^{1,13}$
}

\begin{abstract}
1.-Department of Electronics Engineering, Chang Gung University, Guishan, Taoyuan 333, Taiwan. 2.-Department of Materials Engineering, Ming Chi University of Technology, Taishan, New Taipei City 243, Taiwan. 3.-Department of Otolaryngology-Head and Neck Surgery, Chang Gung Memorial Hospital, Linkou, Taoyuan 333, Taiwan. 4.-Institute of Electro-Optical Engineering, Chang Gung University, Guishan, Taoyuan 333, Taiwan. 5.-Department of Electrical and Electronic Engineering, Chung Cheng Institute of Technology, National Defense University, Daxi, Taoyuan 335, Taiwan. 6.-Green Technology Research Center, Chang Gung University, Guishan, Taoyuan 333, Taiwan. 7.-e-mail: D9828201@cgu.edu.tw. 8.—e-mail: liann@mail.cgu.edu.tw. 9.—e-mail: mjjeng@mail.cgu.edu.tw. 10.—e-mail: D0527103@cgu.edu.tw.11.—e-mail: s943208@gmail.com.12.—e-mail: davenlee15@gmail.com. 13.—e-mail: M0827102@cgu.edu.tw
\end{abstract}

A GaN-based metal-semiconductor-metal varactor with a two-dimensional electron gas (2DEG) layer is proposed and fabricated. The capacitance variation of this fabricated varactor biased at different external voltages is studied and measured, and the frequency-dependent capacitance and resistance of the varactor are simulated by a corresponding empirical formula. A high-frequency protective filter is further constructed and placed under a large pulsedcurrent injection in a malicious electromagnetic interference immunity test. The results show that the proposed GaN-based module can reduce the large pulsed current to an acceptably small level. Thus, the GaN-based 2DEG varactor is an attractive candidate for applications designed to protect the upcoming $5 \mathrm{G}$ high-frequency system from risks such as electrostatic discharge, lightning, and electromagnetic pulses.

Key words: GaN metal-semiconductor-metal varactor, two-dimensional electron gas, malicious electromagnetic interference

\section{INTRODUCTION}

The prevalence of electric vehicles and high-speed cellular phones in modern society is increasing. However, the size of the electronics involved in these devices is continually decreasing. Thus, the probability that these devices will be exposed to high transient current/voltage from the environment is also increasing. For example, the global positioning system (GPS) may encounter harmful electromagnetic pulses. To protect these electronic

(Received January 8, 2020; accepted March 24, 2020;

published online April 10, 2020) systems, which must have precise working properties, from conventional natural or man-made threats, ${ }^{1-5}$ current protection technologies are used. These technologies can be largely classified into three categories: gas discharge tubes (GDTs), ${ }^{6,7}$ metal oxide varistors (MOVs), ${ }^{8-10}$ and transient voltage suppression (TVS) diodes. ${ }^{11}$ GDTs do not have a fast reaction time because of their microsecond-scale ionization of $\mathrm{SF}_{6}$ gases. MOVs have large "in parallel" shunt capacitance and have limited use for high frequencies. TVS diodes have a very fast response time but a small current-sinking ability. Large TVS diodes can improve the current-sinking abilities of these devices, but their parasitic 
capacitance causes issues with high-frequency applications.

On the other hand, two-dimensional electron gas (2DEG) metal-semiconductor-metal (MSM)-structured varactors, which are GaN- or GaAs-based wide-band-gap compound semiconductor devices with very short reaction times, ${ }^{12}$ are highly compatible. By connecting GaN-based 2DEG varactors with an "in series" form, shunt capacitance is no longer a problem, thus benefiting high-frequency applications. Furthermore, these 2DEG varactors represent open circuits at high input power, while the other aforementioned three categories of those types of systems, when connected in parallel at such high input power, may short-circuit. Further, some combination of all three categories of these devices will theoretically increase the efficiency of the protection system. ${ }^{13,14} \mathrm{GaN}$ 2DEG varactors have been used for high-power switching, ${ }^{15}$ photodiodes, ${ }^{16}$ and high-electron-mobility transistor (HEMT) electrostatic discharge (ESD) protection ${ }^{17}$ applications, but studies regarding their use with fast, high-power malicious electromagnetic pulses (MEMI) impulses are lacking. Additionally, fabrication of comprehensive modules for protective purposes and testing of these modules are also lacking. In this study, 2DEG varactor protection modules that use the property of the capacitance variation with respect to the introduced voltage and frequency are studied using a lab-made strip filter and reported upon. The protection capability of this device and module are shown to be compliant with MIL-STD-188-125-2. By comparing the resulting residual currents obtained before and after the high-current double-exponential impulse injections, we confirm that the GaN-biased 2DEG MSM filter module can protect the following circuit from high-impulse damage.

\section{GAN 2DEG VARACTOR FABRICATION}

In this section, we describe the structure of the fabricated GaN 2DEG MSM varactor and its measured low-frequency capacitance under an external bias voltage.

\section{GaN 2DEG Device Structure}

As shown in Fig. 1a, an MSM-2DEG varactor was fabricated using an $\mathrm{Al}_{0.27} \mathrm{Ga}_{0.73} \mathrm{~N} / \mathrm{GaN}$ epitaxial structure on a silicon substrate. ${ }^{18}$ The $\mathrm{Al}_{0.27} \mathrm{Ga}_{0.73} \mathrm{~N} / \mathrm{GaN}$ heterojunction possesses a twodimensional electron gas (2DEG) structure. In Fig. 1b, the metallization of the electrode Schottky contact pads ( $\mathrm{Ti} / \mathrm{Au}$ ) with $90 \mathrm{~nm}$ thickness is shown. In Fig. 1c, the top view of the MSM-2DEG varactor is shown. The separated spacing of the electrodes is $20 \mu \mathrm{m}$.

\section{Capacitance Variation Under an External Voltage}

The GaN 2DEG varactor consists of two Schottky diodes that are connected back-to-back and a 2DEG channel layer as a backside connector. Capacitances are formed in the Schottky depletion zone between the metal and the 2DEG channel, as well as in the lateral depletion zone of the 2DEG. The measured capacitance of the GaN 2DEG varactor under an external bias voltage at $1 \mathrm{MHz}$ is shown in Fig. $1 \mathrm{~d}$. As the external bias voltage increases from $0 \mathrm{~V}$ to $\pm 8 \mathrm{~V}$, the capacitance changes from a maximum to a minimum value. The variation of the capacitance is a symmetrical step function. The cutoff voltage, $V_{\mathrm{T}}$, is around $\pm 4.5 \mathrm{~V}$. When the bias voltage is less than $V_{\mathrm{T}}$, the capacitance approaches its maximum value of $450 \mathrm{pF}\left(C_{\max }\right)$, which is dominated by the capacitance of the Schottky depletion zone between the metal and 2DEG channel $\left(C_{\text {Schottky }}\right)$. When the bias voltage is greater than $V_{\mathrm{T}}$, the depletion zone penetrates the 2DEG channel, creating an additional and small lateral $2 \mathrm{DEG}$ capacitance $\left(C_{2 \mathrm{DEG}}\right)$ in series with $C_{\text {Schottky }}$. As a result, the minimum capacitance of $6.5 \mathrm{pF}\left(C_{\mathrm{min}}\right)$ is equal to $C_{2 \mathrm{DEG}}$. The ratio of the maximum to minimum capacitance is around $65 \sim 70$.

\section{MEASUREMENT RESULTS AND DISCUSSION FOR HIGH-FREQUENCY MEMI IMMUNITY}

The GaN 2DEG capacitance is dependent not only on the biasing voltage, but also on the measuring frequency. Most of the time, we measured the frequency-dependent capacitance of semiconductor devices at low frequency (less than $2 \mathrm{MHz}$ ). For high-frequency applications, we first constructed a simple $50 \Omega$ micro-strip circuit matched with the GaN 2DEG varactor and from which the corresponding high-frequency capacitance was extracted, as shown in Fig. 2a. Next, another micro-strip protective filter with a 2DEG MSM varactor and two high-pass inductors is constructed (as shown in Figs. 6, 7), and its MEMI immunity under a highcurrent impulse source is investigated.

\section{Frequency-Dependent GaN 2DEG Capacitance Determination}

We measured the frequency response and insertion loss of the $50 \Omega$ matched circuit, as depicted in Fig. 2a, for the extraction of the high-frequency capacitance of this system. The capacitance and parallel resistance of the GaN 2DEG varactor diode for frequencies ranging from $1 \mathrm{MHz}$ to $3 \mathrm{GHz}$ were extracted using a network analyzer (shown in Fig. 2b). The maximum capacitance is $450 \mathrm{pF}$, which is the same as that measured in Fig. 1d. The parallel resistance is also dependent upon the 


\begin{tabular}{|c|}
\hline GaN Cap Layer $\sim 2 \mathrm{~nm}$ \\
\hline $\mathrm{Al}_{0.26} \mathrm{Ga}_{0.74} \mathrm{~N}$ Barrier Layer $\sim 24 \mathrm{~nm}$ \\
\hline GaN \\
Buffer Layer $\sim 6 \mu \mathrm{m}$ \\
\hline $\mathrm{Si}(111)$ Substrate $\sim 700 \mu \mathrm{m}$ \\
\hline
\end{tabular}

(a)

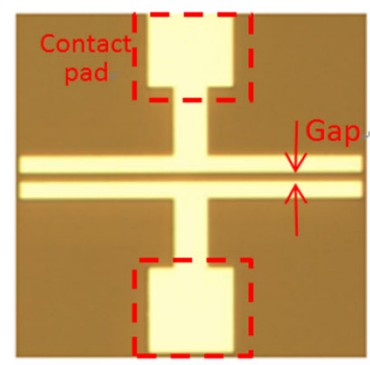

(c)

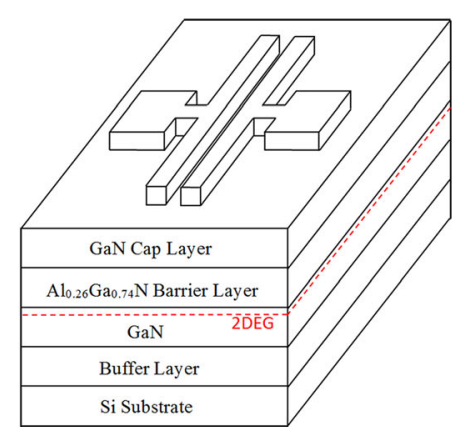

(b)

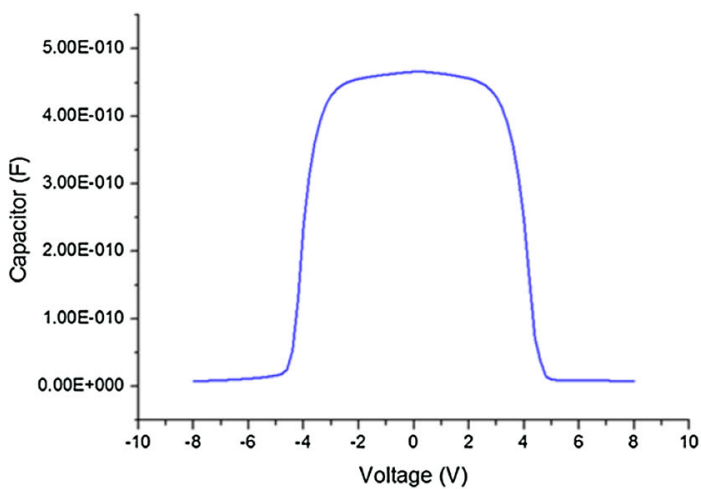

(d)

Fig. 1. (a) AIGaN/GaN MSM-2DEG epitaxial structure. (b) Metallization of the electrode contact pads located on the top of the wafer. (c) Top view of MSM-2DEG varactor with two contacts. (d) Varactor diode under an external voltage measured at $1 \mathrm{MHz}$.

measuring frequencies, and the parallel resistance at $1 \mathrm{MHz}$ is $430 \Omega$. The quality factor, $Q$, of the GaN 2DEG varactor, calculated using the formula $Q=2 \pi f R C$, where $f$ is the frequency, $R$ is the parasitic resistance, and $C$ is the capacitance, is shown in Fig. 2c.

For frequencies below $100 \mathrm{MHz}$, we can use the capacitance variation as our initial value, and we can use a straight line to obtain the capacitance curve, as shown in Fig. 2d. This fitting is achieved using the following empirical formula:

$$
C=C_{0}\left(1-0.5 \log \left(\frac{f}{f_{0}}\right)\right)
$$

where $f_{0}$ and $C_{0}$ are the initial values of the frequency and capacitance, respectively. In this study, $C_{0}=450 \mathrm{pF}$ and $f_{0}=1 \mathrm{MHz}$. This equation demonstrates that the capacitance is a logarithmic function of the frequency.

The conductance or resistance of the GaN 2DEG varactor decreases more rapidly than the capacitance. The resistance variation shown in Fig. 3a is the initial value in determining the straight-line resistance curve. The empirical formula used to obtain the resistance curve is

$$
R=R_{0}\left(\frac{f_{0}}{f}\right)^{0.67},
$$

where $R$ is the resistance, $f$ is the frequency, and $f_{0}$ and $R_{0}$ are the initial values of the frequency and resistance, respectively. In this study, $R_{0}=430 \Omega$ and $f_{0}=1 \mathrm{MHz}$. Figure $3 \mathrm{~b}$ shows the same curves plotted on a linear scale below $100 \mathrm{MHz}$.

These equations therefore demonstrate that after the initial low-frequency values have been determined (from the common $1 \mathrm{MHz} \mathrm{CV}$ meters), the high-frequency $(100 \mathrm{MHz})$-dependent capacitance and resistance of this varactor can be predicted.

By measuring two port parameters of the GaN 2DEG varactor using a network analyzer, we can compare the differences between these values and the fitted formulas. The results are shown in Fig. 4. The insertion loss, S21, and return loss, S11, are shown to be well correlated.

For higher frequencies from $100 \mathrm{MHz}$ to $3 \mathrm{GHz}$, we see that the product of the frequency and capacitance is almost a constant value, i.e., $2 \pi f C=$ constant. Therefore, we can use a straight line to fit the capacitance curve on a log scale, as shown in Fig. 5a and b. The empirical formula is

$$
C=C_{1}\left(\frac{f_{1}}{f}\right)
$$

where the initial values $\left(C_{1}\right.$ and $f_{1}$ for the capacitance and frequency, respectively) can be chosen at 


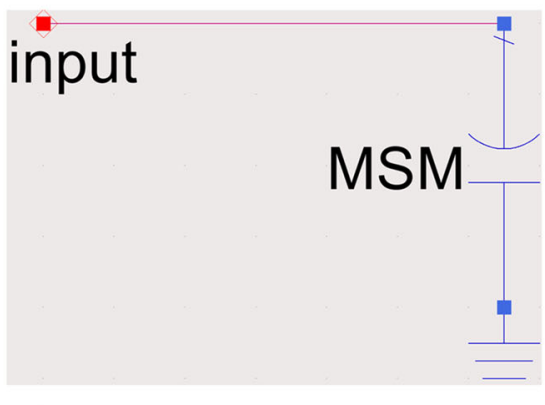

(a)

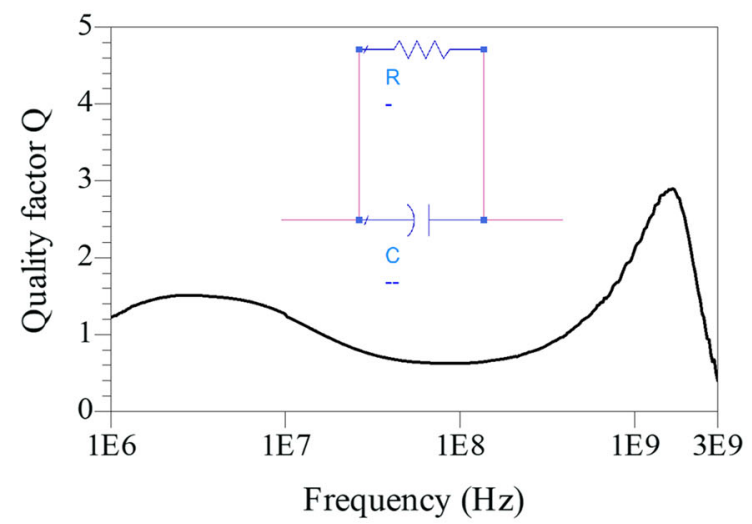

(c)

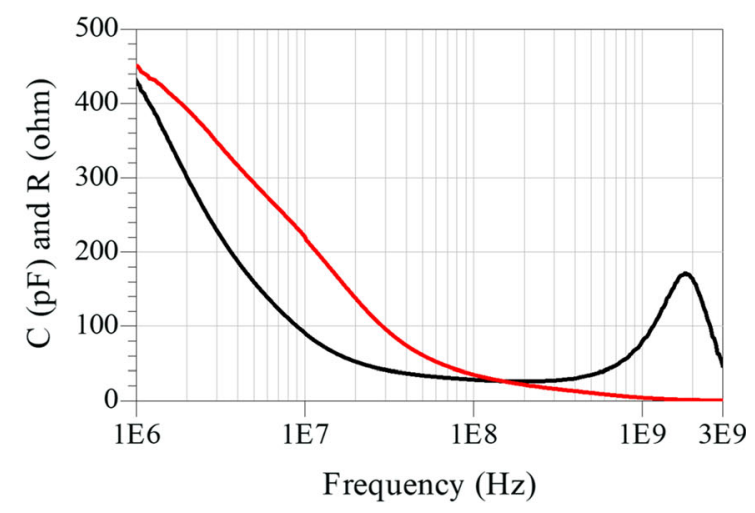

(b)

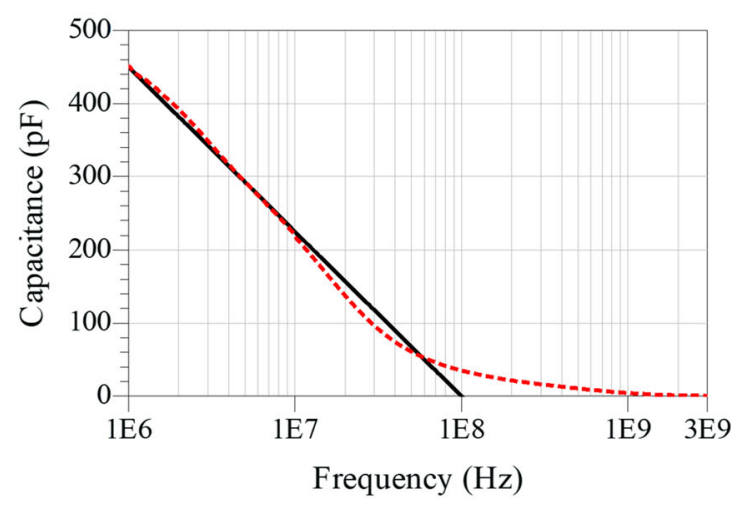

(d)

Fig. 2. (a) The $50 \Omega$ matched micro-strip circuit. (b) Capacitance and resistance of the GaN 2DEG varactor for different frequencies. The red line indicates the capacitance, and the black line indicates the parallel resistance. (c) Quality factor of the GaN 2DEG varactor. (d) Fitted curve of the capacitance of the GaN 2DEG varactor. The dotted line shows the measurement results, while the solid line is the fitted curve (Color figure online).

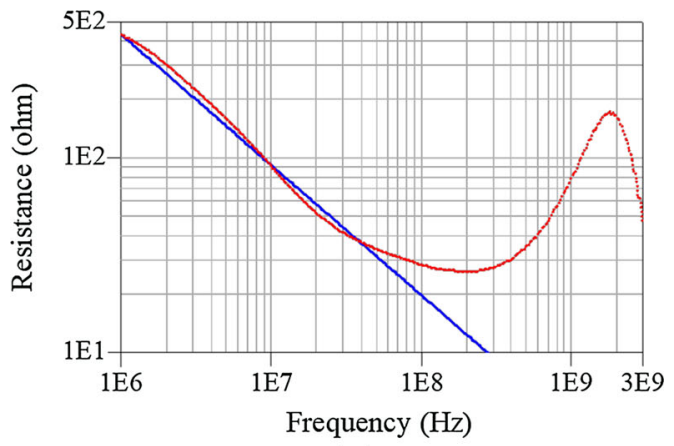

(a)

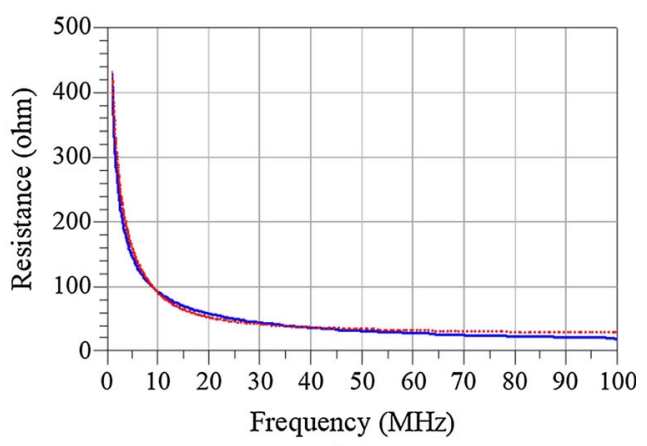

(b)

Fig. 3. Fitted curves of the resistance of the GaN 2DEG varactor. The dotted lines indicate the measurement results, while the solid line is the fitted function. (a) The resistance is fitted using a straight line on a log scale. The plotted frequency ranges from $1 \mathrm{MHz}$ to $3 \mathrm{GHz}$. (b) The fitted resistance curve on a linear scale, over a frequency range below $100 \mathrm{MHz}$.

any point between this interval of the measurement line; in this study, we choose $f_{1}=1.1 \mathrm{GHz}$ and $C_{1}=3 \mathrm{pF}$. The curve correlates well with the experimentally obtained results from $100 \mathrm{MHz}$ to $3 \mathrm{GHz}$, as shown in Fig. 5b.

Figure 5c shows the capacitance of the varactor. All of the values of fabricated GaN 2DEG varactors are below $4 \mathrm{pF}$ for frequencies from $1 \mathrm{GHz}$ to $3 \mathrm{GHz}$.
The dotted line shows the measured values, and the solid line is the fitted curve.

\section{Protection Module Design with GaN 2DEG Varactor}

An ADS schematic diagram of the filter with the GaN 2DEG varactor as a protection module for high-current pulses is shown in Fig. 6. 
The micro-strip filter, as shown in Fig. 7a, was built on a 0.6 -mm-thick FR4 substrate with a relative dielectric constant $(\mathrm{er})$ of 4.3 . The width of the input/output $50 \Omega$ line is $1.14 \mathrm{~mm}$. Two microstrip lines ( $L 1$ and $L 2$ ) act as inductors and are connected to the ground. They are each $17 \mathrm{~mm}$ long and $0.3 \mathrm{~mm}$ wide. The GaN 2DEG varactor is placed at the center.

The prototype of this setup is shown in Fig. $7 \mathrm{~b}$. The lines $L 1$ and $L 2$ are form meander lines to save

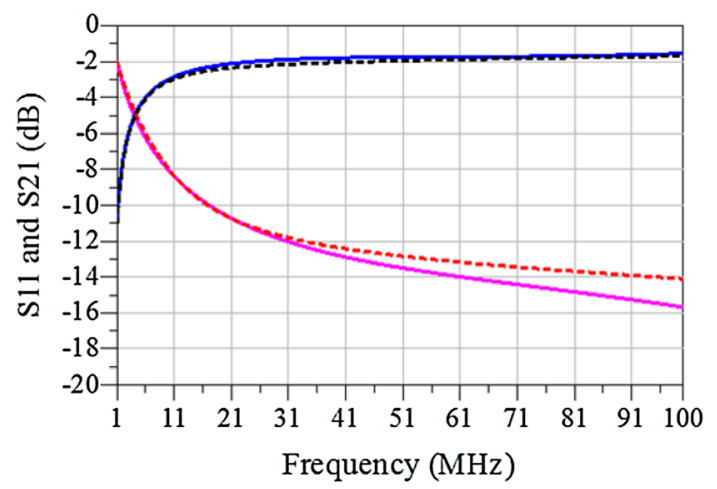

Fig. 4. Two port measurements of the GaN 2DEG varactor. The dotted lines indicate the measurements, while the solid lines are the fitted lines. The insertion loss, S21 (black), and return loss, S11 (red), are plotted on a dB scale. The frequency ranges from $1 \mathrm{MHz}$ to $100 \mathrm{MHz}$ (Color figure online). space. The GaN 2DEG varactor (shown as the white block in Fig. 7b) is placed in the center and is flipchip mounted on the surface.

The filter responses of the simulated and measured data are shown in Fig. 7c. A parallel resistance was added to the simulation to compensate for the insertion loss. The added parallel resistance is $20 \Omega$, which corresponds to a quality factor of 1 . This is confirmed in Fig. 2c. The experimentally obtained center frequency is lower than the corresponding simulation value. This resulted from the $L 1$ and $L 2$ lengths with respect to the ground. The micro-strip lines used in the experimental setup are longer than the simulated lines because of the additional distance due to holes, and this shifts the measured frequencies to lower values.

The fabricated filter is next tested for use with high-current impulse injections. As shown in

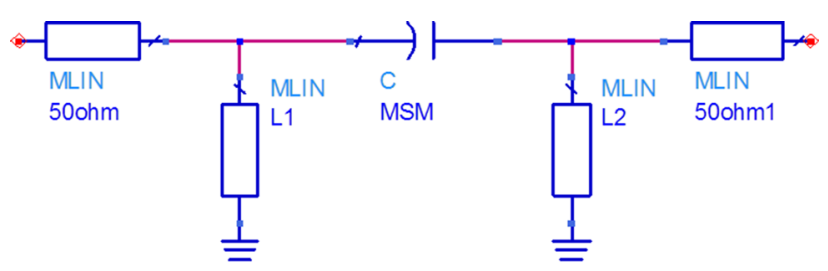

Fig. 6. ADS schematic diagram of the filter. The horizontal line on the right-hand side indicates the input, while the horizontal line on the left-hand side indicates the output.

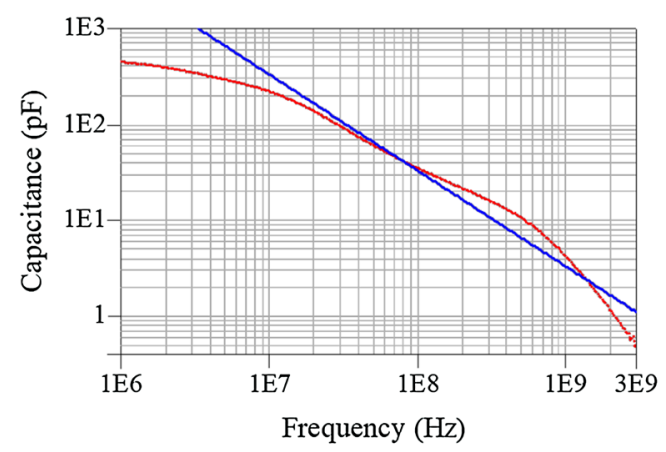

(a)

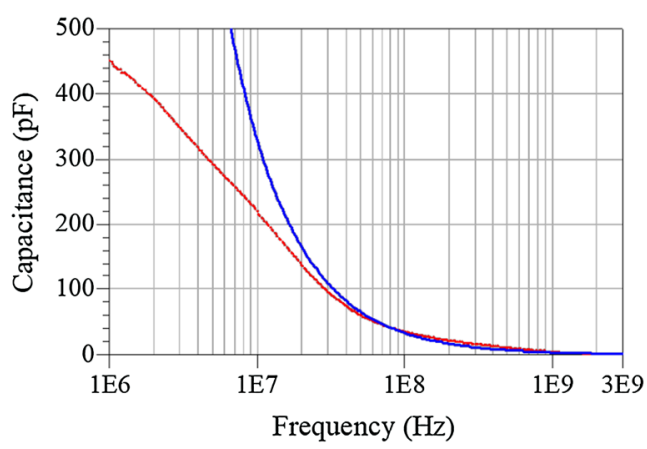

(b)

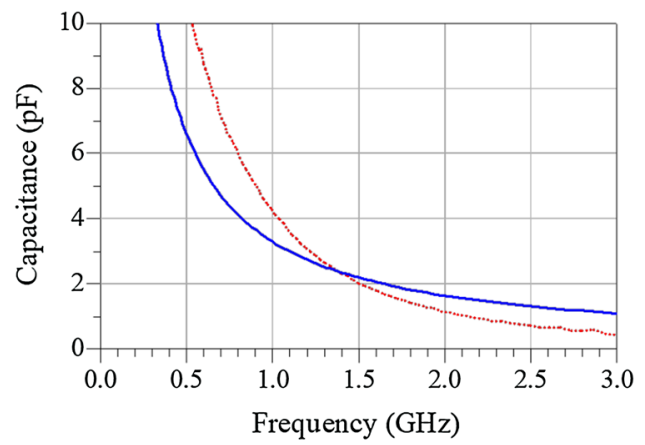

(c)

Fig. 5. Fitted capacitance of the GaN 2DEG varactor. The dotted lines are the measured values, and the solid lines are the fitted curves. (a) The capacitance is fitted using a straight line on a log scale. The plotted frequency ranges from $1 \mathrm{MHz}$ to $3 \mathrm{GHz}$. (b) The same fitted curve, but with a linear-scale $y$-axis. (c) The overall capacitance of the GaN 2DEG varactor below $3 \mathrm{GHz}$. 


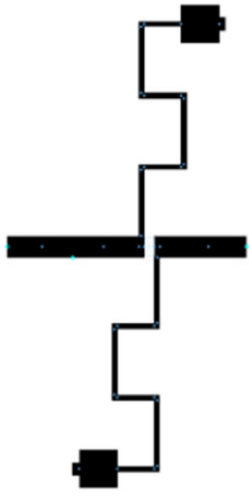

(a)

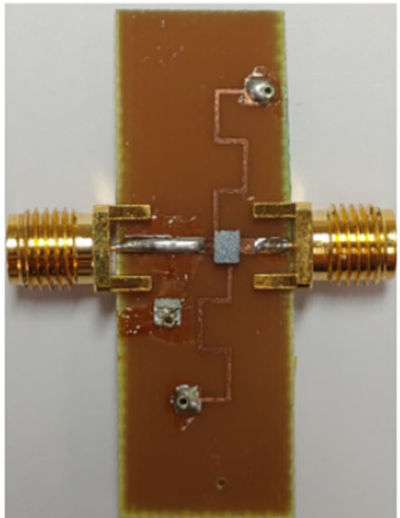

(b)

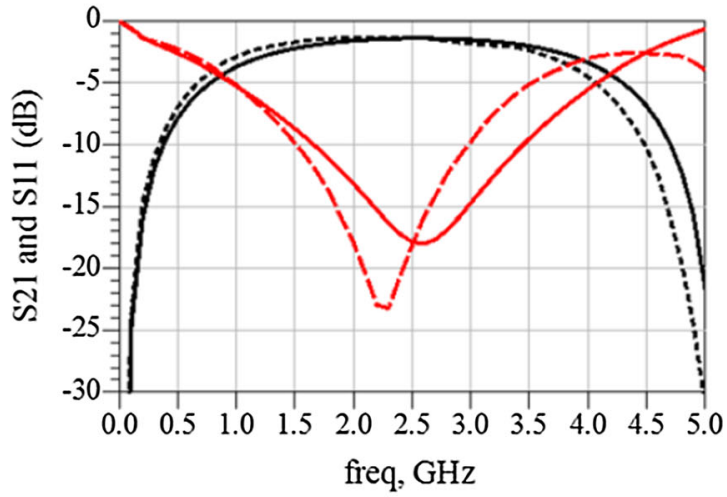

(c)

Fig. 7. Prototype of the filter system. The lines $L 1$ and $L 2$ are form meander lines. (a) Printed circuit board pattern. (b) The prototype of the filter system with the GaN 2DEG varactor and SMA connectors. (c) Filter responses of the simulation (solid lines) and measured data (dotted lines). The insertion loss, S21 (black), and return loss, S11 (red), are plotted on a dB scale. The insertion loss is $1 \mathrm{~dB}$. The $x$-axis shows the frequency over a range from $\mathrm{DC}$ to $5 \mathrm{GHz}$ (Color figure online).

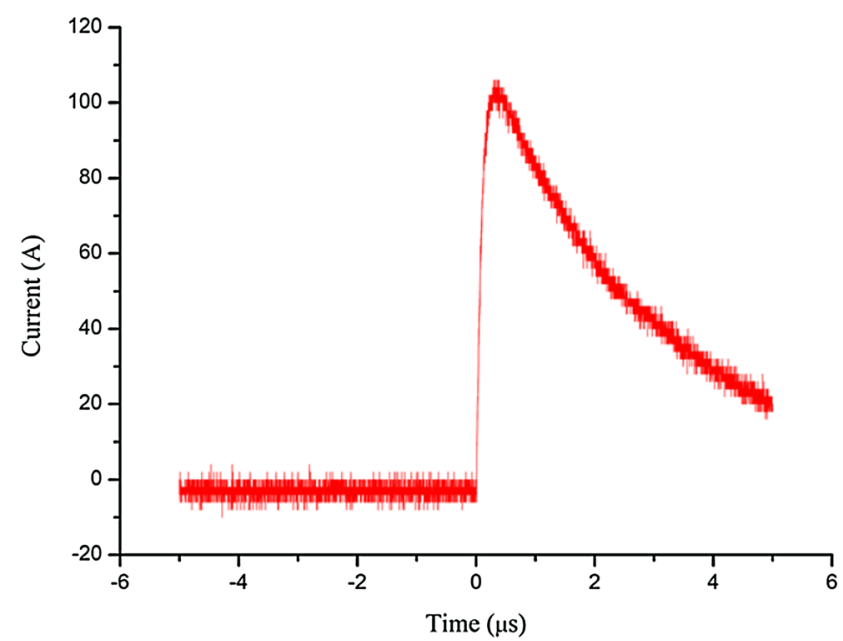

(a)

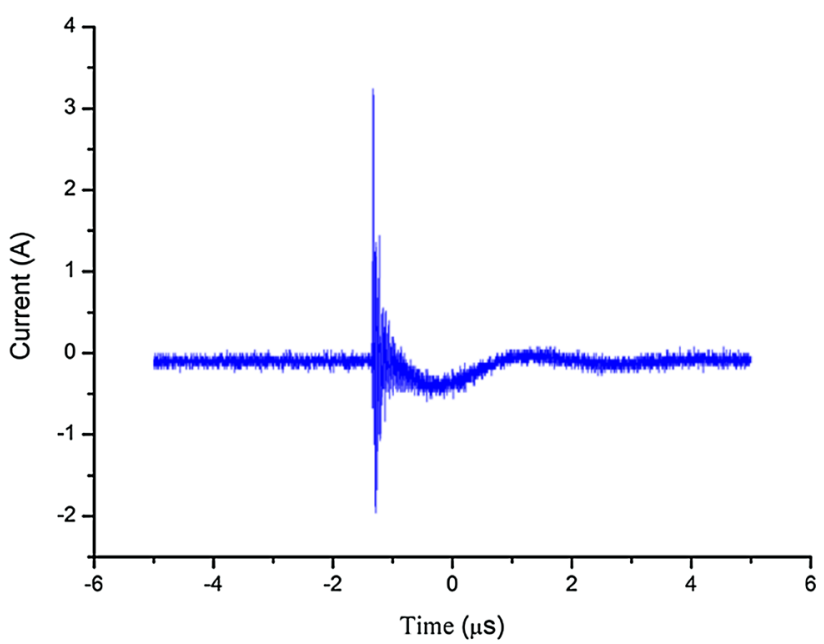

(b)

Fig. 8. The high-current pulse source. (a) The maximum current is $110 \mathrm{~A}$ with a $3 \mu$ s pulse width to a $50 \Omega$ load. (b) Residual output of the highcurrent test. The maximum residual current is $3.5 \mathrm{~A}$.

Fig. 8a, the maximum current of the test source is 110 A with a $3 \mu \mathrm{s}$ pulse width to a $50 \Omega$ load $(\sim 5500 \mathrm{~V})$.

After the high-input-current test, the output to a $50 \Omega$ load is determined, as shown in Fig. 8b. The maximum residual current is as low as $3.5 \mathrm{~A}$. This proves that the GaN-based 2DEG varactor can reduce the high-current impulse injection to a safe level.

After the high-input-current test, the filter is once again measured using a network analyzer. Figure $9 \mathrm{a}$ and $\mathrm{b}$ shows the measurement results of the proposed module. The dotted lines indicate the measurements before the high-current impulse test, while the solid lines show the measurements obtained after this test. The insertion losses (band pass at $1200 \sim 3000 \mathrm{MHz}$ ) are the same, while the return loss shifts by only a minor amount. This shows that the GaN 2DEG MSM varactor can protect the circuit from MEMI- or malicious electromagnetic pulse (MEMP)-related damage and without its own degradation.

\section{CONCLUSIONS}

GaN-based 2DEG MSM varactors were fabricated and tested using positive and negative applied biases. Their capacitance was shown to undergo 


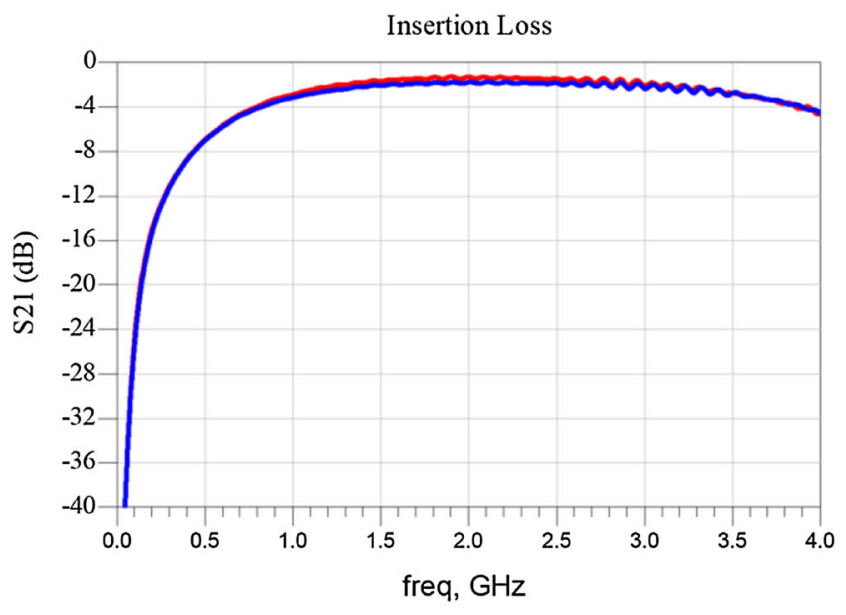

(a)

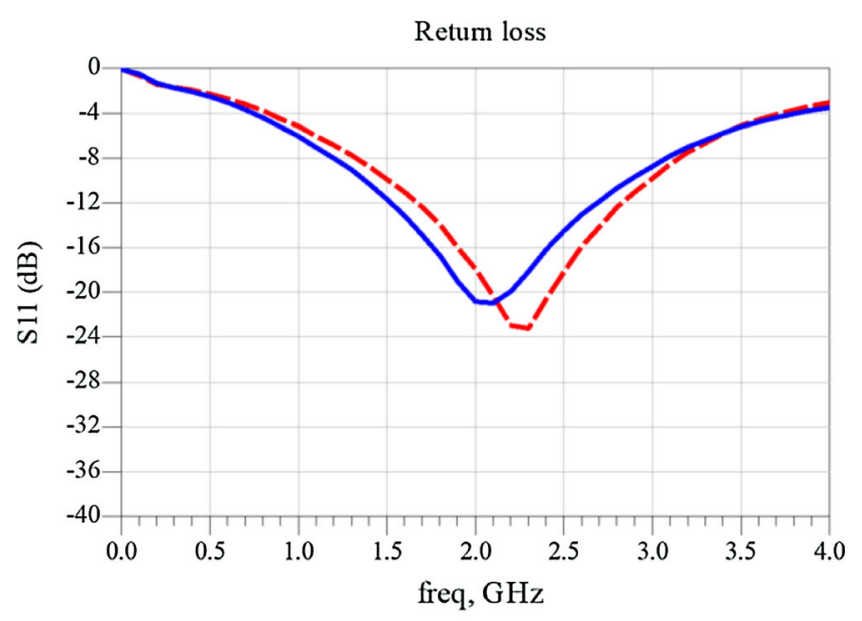

(b)

Fig. 9. Measurements obtained before and after the high-current pulse test. The red line shows the results obtained before the test, and the black line shows those obtained after the test. (a) The insertion loss, S21, of the filter. The results obtained before and after the test are the same. (b) The return loss, $\mathbf{S 1 1}$, of the filter. Only a minor shift between these results is apparent (Color figure online).

an abrupt and fast change under external bias voltage $(\sim 4.5 \mathrm{~V})$. Their frequency-dependent capacitance is also studied and corresponding empirical equations are fitted. These equations demonstrate that after the initial low-frequency values have been determined (from the common $1 \mathrm{MHz} \mathrm{CV}$ meters), the high-frequency (> $100 \mathrm{MHz}$ ) capacitance and resistance of $\mathrm{GaN}$ varactors can be predicted.

The proposed protection module demonstrates the use of this varactor in high-frequency communication systems as an "in series" device to prevent natural MEMP- or MEMI-related damage, in which the proposed varactor is combined with a strip band-pass filter (1200-3000 MHz) to form a protective module. A current injection of $110 \mathrm{~A}$ at a $3 \mu \mathrm{s}$ impulse width was shown to be efficiently suppressed to a value of as low as $3.5 \mathrm{~A}$ by using the proposed module and without degradation on its own performance. This makes the GaN 2DEG MSM varactor based module a useful component for protection from ESD, lightning electromagnetic pulse (LEMP), or even MEMI impulses in the high-frequency range.

\section{ACKNOWLEDGMENTS}

The authors would like to thank Chang Gung University and Taiwan Semiconductor Research Institute and Ministry of Science and Technology, Republic of China, for financially supporting this research.

\section{AUTHOR CONTRIBUTIONS}

L-BC and M-JJ: Conceptualization, C-FS, Y-LH and Z-XD: Data curation, L-BC: Funding acquisition, Y-LH, C-FS, Z-XD and L-BC: Investigation, L-
BC, M-JJ and C-FS: Methodology, L-BC and M-JJ: Project administration, L-BC and M-JJ: Resources, L-BC: Supervision, Y-LH, Y-CL, C-FS, and L-BC: Writing-original draft, C-FS, Y-LH, L-BC and MJJ: Writing—review \& editing.

\section{FUNDING}

This research was funded by Ministry of Science and Technology, Republic of China (MOST 1082221-E-182-022).

\section{CONFLICT OF INTEREST}

The authors declare no conflict of interest. The funders had no role in the design of the study; in the collection, analyses, or interpretation of data; in the writing of the manuscript; or in the decision to publish the results.

\section{OPEN ACCESS}

This article is licensed under a Creative Commons Attribution 4.0 International License, which permits use, sharing, adaptation, distribution and reproduction in any medium or format, as long as you give appropriate credit to the original author(s) and the source, provide a link to the Creative Commons licence, and indicate if changes were made. The images or other third party material in this article are included in the article's Creative Commons licence, unless indicated otherwise in a credit line to the material. If material is not included in the article's Creative Commons licence and your intended use is not permitted by statutory regulation or exceeds the permitted use, you will need to obtain permission directly from the copyright holder. To view a copy of this licence, visit http://creativecom mons.org/licenses/by/4.0/. 


\section{REFERENCES}

1. G. Lugrin, N. Mora, S. Sliman, F. Rachidi, M. Rubinstein, and R. Cherkaoui, in International Symposium on Electromagnetic Compatibility (2013), pp. 24-28.

2. W.A. Radasky, C.E. Baum, and M.W. Wik, IEEE Trans. Electromagn. Compat. 46, 314 (2004).

3. E. Savage and W. Radasky, in IEEE International Symposium on Electromagnetic Compatibility (2012), pp. 317-322.

4. P. Ratna, S. Jain, and J. Chattopadhyay, in Asia-Pacific Microwave Conference (2014), pp. 1175-1177.

5. R. Hao, X. Zhang, H. Gao, H. Wu, J. Cheng, and G.P. Li, Microelectron. J. 84, 1 (2019).

6. Y. Naito, T. Kawabata, S. Yanagawa, and K. Yamamoto, in International Conference on Lightning Protection (ICLP) (2012), pp. 2-7.

7. C.-Y. Tien, L.-B. Chang, P.-Y. Kuei, and C.-F. Chih, in AsiaPacific Symposium on Electromagnetic Compatibility (2015), pp. 467-469.

8. D.T. Khanmiri, R. Ball, C. Mckenzie, and B. Lehman, in IEEE Applied Power Electronics Conference and Exposition (2016), pp. 3038-3042.

9. N. Tsukamoto, in International Conference on Lightning Protection (2014), pp. 620-623.

10. N. Tsukamoto, S. Imato, Y. Baba, and M. Ishii, in 33rd International Conference on Lightning Protection (2016), pp. $1-4$.
11. J. Lepkowski, Avalanche TVS Diode Spice Macro-Models (AND8254/D). (On Semiconductor Website, 2006), https://w ww.onsemi.com/PowerSolutions/extSearch.do?query=AN D8254\&param1=type\&param1_val=document. Accessed May 2006.

12. Y.-C. Ferng, L.-B. Chang, A. Das, C.-C. Lin, C.-Y. Cheng, P.-Y. Kuei, and L. Chow, Jpn. J. Appl. Phys. 51, 124201 (2012).

13. P.L.F. Penalver and da E.S. Braga, TWAS IJCEE. 11, 1 (2017).

14. H. Kijima and K. Murakawa, WSEAS Trans. Power Syst. 7, $60(2012)$

15. G. Simin, A. Koudymov, Z.-J. Yang, V. Adivarahan, J. Yang, and M.A. Khan, IEEE Electron Device Lett. 26, 56 (2005).

16. M. Marso, J. Bernat, M. Wolter, P. Javorka, and A. Fox, in ASDAM 2002 Sniolcnice Castle (2002), pp. 295-298.

17. L.-B. Chang, C.-F. Shih, T.-W. Huang, C.-Y. Tien, and P.-Y. Kuei, in International Symposium on Electromagnetic Compatibility (2014), pp. 717-720.

18. C.-Y. Tien, P.-Y. Kuei, L.-B. Chang, and C.-P. Hsu, J. Electr. Eng. Technol. 10, 1720 (2015).

Publisher's Note Springer Nature remains neutral with regard to jurisdictional claims in published maps and institutional affiliations. 